\title{
A meta-analysis of adjusted hazard ratios from 20 observational studies of bilateral versus single internal thoracic artery coronary artery bypass grafting
}

\author{
Hisato Takagi, MD, PhD, Shin-nosuke Goto, MD, Taku Watanabe, MD, Yusuke Mizuno, MD, \\ Norikazu Kawai, MD, and Takuya Umemoto, MD, PhD
}

\begin{abstract}
Objective: In 2001, a landmark meta-analysis of bilateral internal thoracic artery (BITA) versus single internal thoracic artery (SITA) coronary artery bypass grafting for long-term survival included 7 observational studies (only 3 of which reported adjusted hazard ratios [HRs]) enrolling approximately 16,000 patients. Updating the previous meta-analysis to determine whether BITA grafting reduces long-term mortality relative to SITA grafting, we exclusively abstracte, then combined in a meta-analysis, adjusted (not unadjusted) HRs from observational studies.
\end{abstract}

\begin{abstract}
Methods: MEDLINE and EMBASE were searched until September 2013. Eligible studies were observational studies of BITA versus SITA grafting and reporting an adjusted HR for long-term ( $\geq 4$ years) mortality as an outcome. Meta-regression analyses were performed to determine whether the effects of BITA grafting were modulated by the prespecified factors.

Results: Twenty observational studies enrolling 70,897 patients were identified and included. A pooled analysis suggested a significant reduction in long-term mortality with BITA relative to SITA grafting (HR, 0.80; 95\% confidence interval, 0.77 to 0.84 ). When data from 6 pedicled and 6 skeletonized internal thoracic artery studies were separately pooled, BITA grafting was associated with a statistically significant $26 \%$ and $16 \%$ reduction in mortality relative to SITA grafting, respectively $(P$ for subgroup differences $=.04)$. A meta-regression coefficient was significantly negative for the proportion of men $(-0.00960 ;-0.01806$ to -0.00114$)$.

Conclusions: Based on an updated meta-analysis of exclusive adjusted HRs from 20 observational studies enrolling more than 70,000 patients, BITA grafting seems to significantly reduce long-term mortality. As the proportion of men increases, BITA grafting is more beneficial in reducing mortality. (J Thorac Cardiovasc Surg 2014;148:1282-90)
\end{abstract}

In 2001, a landmark meta-analysis by Taggart and colleagues ${ }^{1}$ of bilateral internal thoracic artery (BITA) versus single internal thoracic artery (SITA) grafting in coronary artery bypass grafting (CABG) was published in the Lancet. The BITA group had significantly better long-term survival than the SITA group (hazard ratio [HR] for death, 0.81; $95 \%$ confidence interval [CI], 0.70 to 0.94 ). The meta-analysis, ${ }^{1}$ however, included only 7 observational studies ${ }^{2-8}$ that had enrolled 15,962 patients and were published in the 1990s. On the one hand, the Cox model was used in an attempt to adjust for the characteristics of the patients (eg, mean age, sex distribution, ventricular function, diabetic status, and so forth) that differed between the 2 groups in

From the Shizuoka Medical Center, Department of Cardiovascular Surgery, Shizuoka, Japan.

Disclosures: Authors have nothing to disclose with regard to commercial support. Received for publication Oct 29, 2013; revisions received Dec 23, 2013; accepted for publication Jan 10, 2014; available ahead of print Feb 9, 2014.

Address for reprints: Hisato Takagi, MD, PhD, Shizuoka Medical Center, Department of Cardiovascular Surgery, 762-1 Nagasawa, Shimizu-cho, Sunto-gun, Shizuoka 411-8611, Japan (E-mail: kfgth973@ybb.ne.jp).

$0022-5223 / \$ 36.00$

Copyright (c) 2014 by The American Association for Thoracic Surgery

http://dx.doi.org/10.1016/j.jtcvs.2014.01.010
3 reports $^{3,5,8}$; on the other hand, no statistical attempts to adjust for the distribution of these variables were made in the other studies. ${ }^{2,4,6,7}$ Furthermore, the largest (enrolling 10,124 patients) study by Lytle and colleagues ${ }^{5}$ had the highest weight $(40.4 \%)$ in the meta-analysis, ${ }^{1}$ and we revealed that exclusion of that study ${ }^{5}$ from the analysis substantively altered the overall result (statistically significant benefit in the BITA group than the SITA group for longterm mortality) to no statistically significant difference (HR, 0.85 ; $95 \%$ CI, 0.68 to 1.05 ; calculated by us) between the 2 groups. Since the previous meta-analysis ${ }^{1}$ was performed, several studies on BITA versus SITA grafting in CABG have been published. In a recent large study published in 2013 by Parsa and colleagues, ${ }^{9}$ which enrolled 17,609 patients (more than the 15,962 patients included in the meta-analysis ${ }^{1}$ ), adjusted mortality was similar (HR, $0.95 ; 95 \%$ CI, 0.83 to 1.08 ) between the BITA and SITA groups. To the best of our knowledge, only 2 randomized controlled trials $^{10,11}$ of BITA versus SITA grafting in CABG have been published until now. In a small trial by Myers and colleagues, ${ }^{10}$ randomizing only 162 patients with a median 7.5-year follow-up, 5-year survival was not different $(96.3 \%$ [ $95 \%$ CI, $92.0 \%$ to $98.7 \%$ ] vs $93.8 \%$ 


\section{Abbreviations and Acronyms \\ BITA = bilateral internal thoracic artery \\ $\mathrm{CABG}=$ coronary artery bypass grafting \\ $\mathrm{CI}=$ confidence interval \\ ITA $=$ internal thoracic artery \\ $\mathrm{OR}=$ odds ratio \\ $\mathrm{RR} \quad=$ relative risk \\ SITA $=$ single internal thoracic artery \\ SWI = sternal wound infection}

[95\% CI, $88.4 \%$ to $97.3 \%$ ]; $P=.39$ ) between the BITA and SITA groups. Another relatively large trial randomizing 3102 patients, the Arterial Revascularization Trial, ${ }^{11}$ also demonstrated similar mortality at only 1 year $(2.5 \%$ for BITA vs $2.3 \%$ for SITA; relative risk [RR], 1.06; $95 \%$ CI, 0.68 to 1.67). Accordingly, evidence for long-term survival in BITA versus SITA grafting in CABG from randomized controlled trials remains insufficient.

Updating the meta-analysis by Taggart and colleagues ${ }^{1}$ to determine whether BITA grafting reduces long-term mortality relative to SITA grafting in CABG, we exclusively abstracted, then combined in a meta-analysis, adjusted (not unadjusted [crude]) risk estimates for long-term mortality from 20 observational studies (more than 70,000 patients were enrolled, which was more than quadruple the number of patients included in the previous metaanalysis ${ }^{1}$ ). Moreover, meta-regression analyses were performed to determine whether the effects of BITA grafting were modulated by prespecified factors.

\section{METHODS}

\section{Search Strategy and Study Selection}

All observational studies of BITA versus SITA grafting that enrolled patients undergoing isolated $\mathrm{CABG}$ and reported adjusted risk estimates for long-term mortality were identified using the same search strategy as in the previous meta-analysis by Taggart and colleagues. ${ }^{1}$ The MEDLINE and EMBASE databases were searched for publications containing the words "internal," "mammary," "thoracic," "single," "unilateral," "bilateral," "multiple," "artery," "arteries," "singly" and in combination, between 1972 (MEDLINE) or 1980 (EMBASE) and September 2013. Two of us (H.T. and S.G.) independently inspected the electronic reports identified by the searches. We included published studies that had at least 100 patients in each group, and had a median (or mean) follow-up of at least 4 years. Only data from the last publication of centers that had produced sequential reports were included. We inspected the references of all studies to identify further studies.

\section{Data Abstraction and Statistical Analysis}

Data regarding detailed inclusion criteria, duration of follow-up, and allcause long-term mortality (adjusted HRs for BITA vs SITA grafting and 95\% CIs) were abstracted from each individual study. All study-specific estimates were combined using inverse variance-weighted averages of logarithmic HRs in both fixed- and random-effects models (primary metaanalysis). Between-study heterogeneity was analyzed by means of standard $\chi^{2}$ tests. Where nonsignificant statistical heterogeneity was identified, the fixed-effects estimate was used preferentially as the summary measure. Sensitivity analyses were performed to assess the contribution of each study to the pooled estimate by excluding individual studies 1 at a time and recalculating the pooled HR estimates for the remaining studies (1-studyremoved meta-analysis). To assess the impact of differential internal thoracic artery (ITA) harvesting techniques among the studies on the pooled estimate, the effects of BITA grafting on long-term mortality were explored separately in studies using the pedicled and skeletonized ITA. Publication bias was assessed graphically using a funnel plot and mathematically using adjusted rank correlation and linear regression tests. Mixed-effects (unrestricted maximum likelihood) meta-regression analyses were performed to determine whether the effects of BITA grafting were modulated by prespecified factors: that is, the mean length of follow-up or age (years), and proportion of men or diabetes (\%). Meta-regression graphs depict the effect of BITA grafting on the outcome (plotted as $\log \mathrm{HR}$ on the y-axis) as a function of a given factor (plotted as a mean or proportion of that factor on the x-axis). Meta-regression coefficients (slopes of the meta-regression line) show the estimated increase in $\log H \mathrm{R}$ per unit increase in the covariate. Because $\log \mathrm{HR}>0$ corresponds to $\mathrm{HR}>1$ and $\log \mathrm{HR}<0$ corresponds to $\mathrm{HR}<1$, a negative coefficient indicates that as a given factor increases, the HR decreases; that is, BITA grafting is more beneficial in reducing the outcome of interest. All analyses were conducted using Review Manager version 5.2 (Nordic Cochrane Centre, Copenhagen, Denmark) and Comprehensive Meta-Analysis version 2 (Biostat, Englewood, NJ).

\section{RESULTS}

\section{Search Results}

Our comprehensive search identified 20 observational studies $^{3,8,9,12-28}$ of BITA versus SITA grafting that enrolled patients undergoing $\mathrm{CABG}$ and reported adjusted risk estimates for long-term mortality. In total, our metaanalysis included data on 70,897 patients undergoing CABG assigned to BITA or SITA grafting. The baseline characteristics for the patients enrolled in each study are summarized in Table 1. The most notable difference in some studies ${ }^{13,19,28}$ from the others was the criteria for enrollment of patients. The studies by Bonacchi and colleagues, ${ }^{13}$ Di Mauro and colleagues, ${ }^{15}$ Joo and colleagues, ${ }^{19}$ Kinoshita and colleagues, ${ }^{21}$ Navia and colleagues, ${ }^{25}$ and Toumpoulis and colleagues ${ }^{28}$ exclusively enrolled patients undergoing nonelective $\mathrm{CABG},{ }^{13}$ those aged less than 70 years, ${ }^{15}$ those undergoing off-pump $\mathrm{CABG},{ }^{19}$ those aged more than 70 years,${ }^{21}$ those undergoing total arterial off-pump $\mathrm{CABG},{ }^{25}$ and those with diabetes, ${ }^{28}$ respectively. Despite the noted heterogeneity in design among studies, there was sufficient similarity between the populations and the hypotheses to merit inclusion of all 20 studies in the quantitative meta-analysis. The most dissimilar studies (Bonacchi and colleagues, 2006, ${ }^{13}$ Di Mauro and colleagues, 2005, ${ }^{15}$ Joo and colleagues, 2012, ${ }^{19}$ Kinoshita and colleagues, 2012, ${ }^{21}$ Navia and colleagues, $2013,{ }^{25}$ and Toumpoulis and colleagues, $2006^{28}$ ) were sequentially eliminated in sensitivity analyses to assess their impact on the pooled effect estimate.

\section{Primary Meta-Analysis}

A pooled analysis of all 20 studies demonstrated a statistically significant $20 \%$ reduction in long-term mortality 
TABLE 1. Trial design and patient characteristics

\begin{tabular}{|c|c|c|c|c|c|c|c|}
\hline \multicolumn{3}{|c|}{ Study } & \multicolumn{2}{|c|}{ Criteria } & \multirow[b]{2}{*}{ ITA harvesting } & \multirow{2}{*}{$\begin{array}{c}\text { Adjustment } \\
\text { method }\end{array}$} & \multirow[b]{2}{*}{ Subgroup } \\
\hline First author, year & Institute & Period & Inclusion & Exclusion & & & \\
\hline $\begin{array}{l}\text { APPROACH } \\
\qquad \text { (Kieser), } 2011^{12}\end{array}$ & $\begin{array}{l}\text { [The Province of } \\
\text { Alberta, Canada] }\end{array}$ & $\begin{array}{l}\text { April } 1995 \text { to } \\
\text { March } 2008\end{array}$ & Isolated primary $\mathrm{CABG}$ & Non-Alberta residents & NA & MCPHR & \\
\hline Bonacchi, $2006^{13}$ & University of Florence & $1997-2003$ & $\begin{array}{l}\text { Nonelective } \mathrm{CABG} \text { for } \\
\text { unstable angina }\end{array}$ & - & Skeletonized & MCPHR & \\
\hline Buxton, $1998^{3}$ & $\begin{array}{l}\text { Austin and Repatriation } \\
\text { Medical Centre }\end{array}$ & 1985-1995 & $\begin{array}{l}\text { [Including urgent/ } \\
\text { emergency CABG] }\end{array}$ & $\begin{array}{l}\text { Severe or poorly } \\
\text { controlled type } 1 \\
\text { diabetes, morbid } \\
\text { obesity, severe } \\
\text { COAD, and } \\
\text { cardiogenic shock } \\
\text { for BITA }\end{array}$ & Pedicled & MCPHR & \\
\hline Carrier, $2009^{14}$ & Montreal Heart Institute & $1995-2007$ & Isolated primary $\mathrm{CABG}$ & Redo CABG & Pedicled & MCPHR & $\begin{array}{l}\text { Without statin } \\
\text { With statin }\end{array}$ \\
\hline Di Mauro, $2005^{15}$ & $\begin{array}{l}\text { "G. D’Annunzio" } \\
\text { University; University } \\
\text { Hospital, Torino }\end{array}$ & $\begin{array}{r}\text { September } 1986 \text { to } \\
\text { December } 1999\end{array}$ & $\begin{array}{l}\text { Primary CABG; }<70 \\
\text { years }\end{array}$ & - & Pedicled skeletonized & $\begin{array}{l}\text { MCPHR in } \\
\text { PS-matched } \\
\text { patients }\end{array}$ & \\
\hline Endo, $2001^{16}$ & $\begin{array}{l}\text { Tokyo Women's Medical } \\
\text { University }\end{array}$ & $\begin{array}{l}\text { April } 1985 \text { to } \\
\quad \text { March } 1998\end{array}$ & $\begin{array}{l}\text { Elective, isolated primary } \\
\text { CABG [including children } \\
\text { with Kawasaki disease] }\end{array}$ & Long-term dialysis & Skeletonized & MCPHR & \\
\hline Glineur, $2012^{17}$ & $\begin{array}{l}\text { Université Catholique } \\
\text { de Louvain }\end{array}$ & $\begin{array}{l}\text { July } 1985 \text { to } \\
\quad \text { November } 1995\end{array}$ & Isolated CABG & - & NA & $\begin{array}{l}\text { MCPHR using PS } \\
\text { strata }\end{array}$ & \\
\hline Grau, $2012^{18}$ & $\begin{array}{l}\text { Valley Heart and } \\
\text { Vascular Institute }\end{array}$ & $1994-2010$ & Isolated CABG & Redo CABG; using RA & Routinely pedicled & $\begin{array}{l}\text { MCPHR in } \\
\text { PS-matched } \\
\text { patients }\end{array}$ & \\
\hline Joo, $2012^{19}$ & $\begin{array}{l}\text { Yonsei Cardiovascular } \\
\text { Hospital }\end{array}$ & 2000-2009 & Isolated $\mathrm{OPCAB}$ & - & Semiskeletonized & PS matching & \\
\hline Kelly, $2012^{20}$ & $\begin{array}{l}\text { Queen Elizabeth II } \\
\text { Health Sciences } \\
\text { Center }\end{array}$ & $1995-2009$ & Isolated primary $\mathrm{CABG}$ & Redo CABG & Pedicled & $\begin{array}{l}\text { Nonparsimonious } \\
\text { CPHR including } \\
\text { PS quintiles }\end{array}$ & \\
\hline Kinoshita, $2012^{21}$ & $\begin{array}{l}\text { Shiga University of } \\
\text { Medical Science }\end{array}$ & $2002-2010$ & Isolated CABG; $>70$ years & $\begin{array}{l}\text { Emergency CABG } \\
\text { with PCBS }\end{array}$ & Skeletonized & $\begin{array}{l}\text { MCPHR in } \\
\text { PS-matched } \\
\text { patients }\end{array}$ & \\
\hline Kurlansky, $2010^{22}$ & $\begin{array}{l}\text { Florida Heart Research } \\
\text { Institute }\end{array}$ & $\begin{array}{l}\text { February } 1972 \text { to } \\
\quad \text { May } 1994\end{array}$ & Isolated CABG & - & Skeletonized & MCPHR & \\
\hline Locker, $2012^{23}$ & Mayo Clinic & 1993-2009 & Isolated primary $\mathrm{CABG}$ & - & Pedicled skeletonized & Stepwise MCPHR & $\begin{array}{l}\text { BITA only* } \\
\text { BITA/RA } \\
\text { BITA/SV }\end{array}$ \\
\hline Lytle, $2004^{24}$ & $\begin{array}{l}\text { Cleveland Clinic } \\
\text { Foundation }\end{array}$ & 1971-1989 & $\begin{array}{l}\text { Nonforeign; nonemergency, } \\
\text { isolated primary CABG }\end{array}$ & $\begin{array}{l}\text { Using non-ITA arterial } \\
\text { grafts }\end{array}$ & NA & PS matching & \\
\hline Navia, $2013^{25}$ & $\begin{array}{l}\text { Institute Cardiovascular } \\
\text { of Buenos Aires }\end{array}$ & $\begin{array}{c}\text { January } 2003 \text { to } \\
\text { May } 2011\end{array}$ & $\begin{array}{l}\text { Urgent/elective total arterial } \\
\text { OPCAB with LITA and } \\
\text { RITA or RA }\end{array}$ & - & Mostly skeletonized & PS matching & \\
\hline Parsa, $2013^{9}$ & $\begin{array}{l}\text { Duke University Medical } \\
\text { Center }\end{array}$ & 1984-2009 & Isolated CABG & - & NA & MCPHR & \\
\hline Pick, $1997^{8}$ & Mayo Clinic & $\begin{array}{c}\text { January } 1984 \text { to } \\
\text { May } 1986\end{array}$ & Isolated CABG & $\begin{array}{c}\text { Redo CABG; alternate } \\
\text { venous or arterial } \\
\text { conduits; multiple } \\
\text { endarterectomies }\end{array}$ & Pedicled & MCPHR & \\
\hline Puskas, $2012^{26}$ & Emory University & $2002-2010$ & Isolated primary $\mathrm{CABG}$ & Emergency CABG & Pedicled/skeletonized & MCPHR using PS & \\
\hline Stevens, $2004^{27}$ & Montreal Heart Institute & 1985-1995 & $\begin{array}{l}\text { Isolated primary } \mathrm{CABG} \text { with } \\
\geq 3 \text { grafts }\end{array}$ & $\begin{array}{l}\text { Redo CABG; using } \\
\text { RGEA }\end{array}$ & Pedicled & $\begin{array}{l}\text { Stepwise MCPHR } \\
\text { including PS }\end{array}$ & \\
\hline Toumpoulis, $2006^{28}$ & $\begin{array}{l}\text { St. Luke's-Roosevelt } \\
\text { Hospital Center and } \\
\text { Columbia University }\end{array}$ & $\begin{array}{c}\text { January } 1992 \text { to } \\
\text { March } 2002\end{array}$ & Diabetes; isolated CABG & - & NA & $\begin{array}{l}\text { Backward stepwise } \\
\text { MCPHR in } \\
\text { PS-matched } \\
\text { patients }\end{array}$ & \\
\hline
\end{tabular}

APPROACH, Alberta Provincial Project for Outcomes Assessment in Coronary Heart Disease database; ITA, internal thoracic artery; BITA, bilateral internal thoracic artery; SITA, single internal thoracic artery; $S D$, standard deviation; $C A B G$, coronary artery bypass grafting; $N A$, not available; $M C P H R$, multivariable Cox proportional hazards regression; $E F$, ejection fraction; $C O A D$, chronic-obstructive-airway disease; $P S$, propensity score; $R A$, radial artery; $O P C A B$, off-pump coronary artery bypass; $C P H R$, Cox proportional hazards regression; $S V$, saphenous vein; $L I T A$, left internal thoracic artery; RITA, right internal thoracic artery; $P C B S$, percutaneous cardiopulmonary bypass support; $L V D$, left ventricular dysfunction; RGEA, right gastroepiploic artery. *Composite-T grafting. †Including other multiple arterial grafting $(\mathrm{n}=180)$.

with BITA relative to SITA grafting in the fixed-effects model (HR, 0.80; 95\% CI, 0.77 to $0.84 ; P<.00001$; Figure 1). There was minimal study heterogeneity of the results $(P=.30)$ and, accordingly, little difference in the pooled result from random-effects modeling (HR, 0.80; $95 \%$ CI, 0.76 to $0.84 ; P<.00001$ ). 
TABLE 1. Continued

\begin{tabular}{|c|c|c|c|c|c|c|c|c|c|c|c|c|c|}
\hline \multicolumn{2}{|c|}{$\begin{array}{c}\begin{array}{c}\text { Number of } \\
\text { patients }\end{array} \\
\end{array}$} & \multicolumn{2}{|c|}{$\begin{array}{l}\text { Mean }( \pm \text { SD) length } \\
\text { of follow-up (years) }\end{array}$} & \multicolumn{2}{|c|}{ Mean $( \pm \mathrm{SD})$ age (years) } & \multicolumn{2}{|c|}{ Men $(\%)$} & \multicolumn{4}{|c|}{ Ventricular function } & \multicolumn{2}{|c|}{ Diabetes (\%) } \\
\hline BITA & SITA & BITA & SITA & BITA & SITA & BITA & SITA & & fication & BITA & SITA & BITA & SITA \\
\hline 1038 & 4029 & $6.4 \pm 3.2$ & $7.1 \pm 3.4$ & $58.0 \pm 9.1$ & $67.6 \pm 9.5$ & & & $\mathrm{EF}$ & $\begin{array}{c}<0.20 \\
0.20-0.34 \\
0.35-0.50 \\
>0.50\end{array}$ & $\begin{array}{r}0.4 \% \\
3.4 \% \\
20.5 \% \\
71.0 \%\end{array}$ & $\begin{array}{r}0.4 \% \\
5.4 \% \\
22.2 \% \\
66.5 \%\end{array}$ & 27.8 & 26.2 \\
\hline 320 & 332 & & & $59 \pm 14$ & $63 \pm 11$ & 81.9 & 80.1 & EF & $<0.35$ & $19.1 \%$ & $20.8 \%$ & 30.0 & 33.7 \\
\hline 1269 & 1557 & & & $58.6 \pm 9$ & $64.9 \pm 9$ & 89.4 & 78.0 & $\mathrm{EF}$ & $<0.50$ & $4.9 \%$ & $24.2 \%$ & 6.8 & 19.9 \\
\hline
\end{tabular}

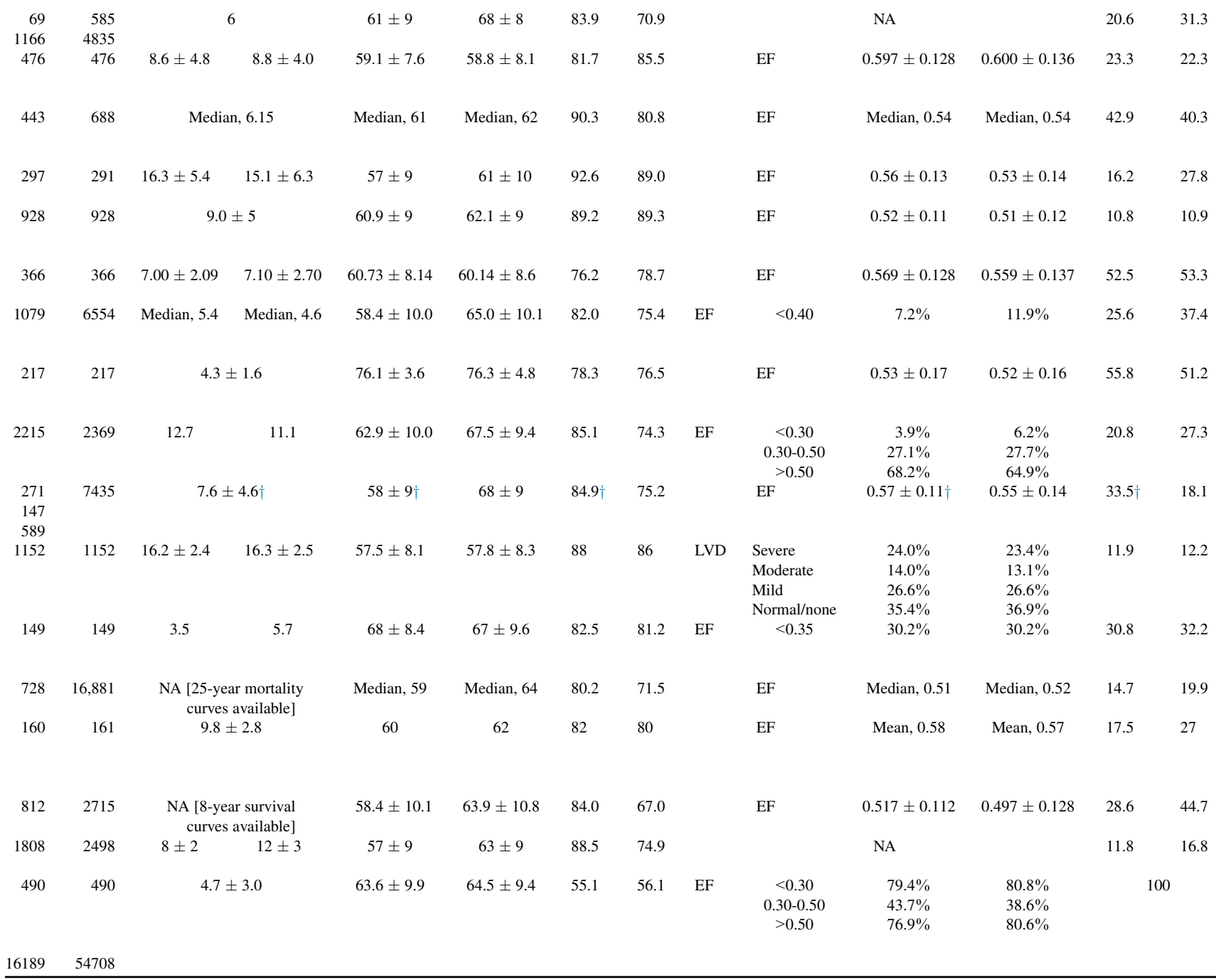

\section{Sensitivity Analyses}

To assess the impact of qualitative heterogeneity in the study design and patient selection on the pooled effect estimate, we performed several sensitivity analyses. First, we sequentially excluded Bonacchi and colleagues, $2006,{ }^{13}$ Di Mauro and colleagues, 2005, ${ }^{15}$ Kinoshita and 


\begin{tabular}{|c|c|c|c|c|c|c|c|c|}
\hline Study or Subgroup & log[Hazard Ratio] & SE & $\begin{array}{l}\text { BITA } \\
\text { Total }\end{array}$ & $\begin{array}{l}\text { SITA } \\
\text { Total }\end{array}$ & Weight & $\begin{array}{l}\text { Hazard Ratio } \\
\text { IV, Fixed, } 95 \% \mathrm{CI}\end{array}$ & $\begin{array}{l}\text { Hazarc } \\
\text { IV, Fixed }\end{array}$ & $\begin{array}{l}\text { Ratio } \\
\text { I, } 95 \% \mathrm{Cl}\end{array}$ \\
\hline APPROACH 2011 & -0.1424 & 0.1166 & 1038 & 4029 & $3.9 \%$ & $0.87[0.69,1.09]$ & & F \\
\hline Bonacchi 2006 & -0.4055 & 0.6551 & 320 & 332 & $0.1 \%$ & $0.67[0.18,2.41]$ & & \\
\hline Buxton 1998 & -0.3415 & 0.1256 & 1269 & 1557 & $3.4 \%$ & $0.71[0.56,0.91]$ & & \\
\hline Carrier 2009 , statin (+) & -0.0781 & 0.1562 & 1166 & 4835 & $2.2 \%$ & $0.92[0.68,1.26]$ & & \\
\hline Carrier 2009 , statin (-) & -0.4368 & 0.1197 & 69 & 585 & $3.7 \%$ & $0.65[0.51,0.82]$ & & \\
\hline Di Mauro 2005 & -0.5306 & 0.2349 & 476 & 476 & $1.0 \%$ & $0.59[0.37,0.93]$ & & \\
\hline Endo 2001 & -0.0502 & 0.1787 & 443 & 688 & $1.7 \%$ & $0.95[0.67,1.35]$ & & \\
\hline Glineur 2012 & -0.298 & 0.1259 & 297 & 291 & $3.4 \%$ & $0.74[0.58,0.95]$ & & \\
\hline Grau 2012 & -0.3955 & 0.1126 & 928 & 928 & $4.2 \%$ & $0.67[0.54,0.84]$ & & \\
\hline Joo 2012 & -0.0125 & 0.2648 & 366 & 366 & $0.8 \%$ & $0.99[0.59,1.66]$ & & \\
\hline Kelly 2012 & -0.2008 & 0.1004 & 1079 & 6554 & $5.3 \%$ & $0.82[0.67,1.00]$ & & \\
\hline Kinoshita 2012 & -0.5906 & 0.2962 & 217 & 217 & $0.6 \%$ & $0.55[0.31,0.99]$ & & \\
\hline Kurlansky 2010 & -0.1778 & 0.0426 & 2215 & 2369 & $29.5 \%$ & $0.84[0.77,0.91]$ & $\rightarrow-$ & \\
\hline Locker 2012 , BITA only & -0.2247 & 0.1904 & 271 & 0 & $1.5 \%$ & $0.80[0.55,1.16]$ & & \\
\hline Locker 2012, BITA/RA & -0.1434 & 0.2412 & 147 & 0 & $0.9 \%$ & $0.87[0.54,1.39]$ & & \\
\hline Locker 2012, BITA/SV & -0.312 & 0.1276 & 589 & 7435 & $3.3 \%$ & $0.73[0.57,0.94]$ & & \\
\hline Lytle 2004 & -0.2739 & 0.0704 & 1152 & 1152 & $10.8 \%$ & $0.76[0.66,0.87]$ & & \\
\hline Navia 2013 & -0.0194 & 0.343 & 149 & 149 & $0.5 \%$ & $0.98[0.50,1.92]$ & & \\
\hline Parsa 2013 & -0.0547 & 0.0672 & 728 & 16881 & $11.9 \%$ & $0.95[0.83,1.08]$ & & - \\
\hline Pick 1997 & -0.2002 & 0.2489 & 160 & 161 & $0.9 \%$ & $0.82[0.50,1.33]$ & & \\
\hline Puskas 2012 & -0.4309 & 0.1546 & 812 & 2715 & $2.2 \%$ & $0.65[0.48,0.88]$ & & \\
\hline Stevens 2004 & -0.3081 & 0.1034 & 1808 & 2498 & $5.0 \%$ & $0.73[0.60,0.90]$ & & \\
\hline Toumpoulis 2006 & -0.12 & 0.1281 & 490 & 490 & $3.3 \%$ & $0.89[0.69,1.14]$ & & \\
\hline Total $(95 \% \mathrm{Cl})$ & & & 16189 & 54708 & $100.0 \%$ & $0.80[0.77,0.84]$ & & \\
\hline \multicolumn{7}{|c|}{$\begin{array}{l}\text { Heterogeneity: } \mathrm{Chi}^{2}=25.01, \mathrm{df}=22(\mathrm{P}=0.30) ; \mathrm{I}^{2}=12 \% \\
\text { Test for overall effect: } \mathrm{Z}=9.38(\mathrm{P}<0.00001)\end{array}$} & \begin{tabular}{|cc}
1 \\
0.5 & 0.7 \\
Favours
\end{tabular} & 1 Favo \\
\hline
\end{tabular}

FIGURE 1. Forest plot of adjusted hazard ratios for long-term mortality among patients assigned to bilateral versus single internal thoracic artery grafting. BITA, Bilateral internal thoracic artery; SITA, single internal thoracic artery grafting; SE, standard error; $C I$, confidence interval; $I V$, inverse variance; $R A$, radial artery; $S V$, saphenous vein.

colleagues, 2012, ${ }^{21}$ and Toumpoulis and colleagues, 2006, ${ }^{28}$ which exclusively enrolled patients undergoing nonelective CABG for unstable angina, ${ }^{13}$ those aged less than 70 years, ${ }^{15}$ those aged more than 70 years, ${ }^{21}$ and those with diabetes, ${ }^{28}$ respectively; combining the remaining 19 studies still generated a statistically significant result favoring BITA grafting (fixed-effects HR for exclusion of Bonacchi and colleagues, $2006,{ }^{13} 0.81 ; 95 \% \mathrm{CI}, 0.77$ to 0.84 ; $P<.00001$; fixed-effects HR for exclusion of Di Mauro and colleagues, $2005,{ }^{15} 0.81 ; 95 \% \mathrm{CI}, 0.77$ to 0.84 ; $P<.00001$; fixed-effects HR for exclusion of Kinoshita and colleagues, $2012,{ }^{21} 0.81 ; 95 \% \mathrm{CI}, 0.77$ to 0.84 ; $P<.00001$; fixed-effects HR for exclusion of Toumpoulis and colleagues, $2006,{ }^{28} 0.80 ; 95 \% \mathrm{CI}, 0.77$ to 0.84 ; $P<.00001)$. Second, we excluded both Joo and colleagues, $2012^{19}$ and Navia and colleagues, $2013,{ }^{25}$ both of which exclusively enrolled patients undergoing off-pump CABG. ${ }^{19,25}$ Without them, there was still a statistically significant benefit for BITA grafting in pooled analysis of the remaining 18 studies (fixed-effects HR, 0.80; 95\% CI, 0.77 to $0.84 ; P<.00001)$. In general, exclusion of any single study from the analysis did not substantively alter the overall result of our analysis (1-study-removed metaanalysis; Figure 2). When data from 6 pedicled $^{3,8,14,18,20,27}$ (enrolling 23,597 patients) and 6 skeletonized ITA studies $^{13,16,19,21,22,25}$ (enrolling 7,831 patients) were separately pooled using the fixed-effects model, BITA grafting was associated with a statistically significant $26 \% \quad$ (HR, $0.74 ; 95 \%$ CI, 0.68 to $0.81 ; P$ for effect $<.00001 ; P$ for heterogeneity $=.51)$ and $16 \%(\mathrm{HR}$, $0.84 ; 95 \%$ CI, 0.78 to $0.91 ; P$ for effect $<.0001 ; P$ for heterogeneity $=.65)$ reduction in long-term mortality relative to SITA grafting ( $P$ for subgroup differences $=.04$; Figure 3).

\section{Publication Bias}

To assess publication bias we generated a funnel plot of the logarithm of effect size versus the precision (reciprocal of the standard error) for each study (data not shown). There was no evidence of significant publication bias (2-tailed $P$ with continuity correction $=.95787$ by the adjusted rank correlation test and 2-tailed $P=.21818$ by the linear regression test).

\section{Meta-Regression Analyses}

Meta-regression coefficients were not statistically significant for the mean length of follow-up (coefficient, -0.00194 ; $95 \% \mathrm{CI},-0.01526$ to $0.01137 ; P=.77472)$, mean age (coefficient, $0.01087 ; 95 \% \mathrm{CI},-0.00448$ to 0.02622 ; $P=.16528$ ), and proportion of diabetes (coefficient, $-0.00167 ; 95 \% \mathrm{CI},-0.00137$ to $0.00471 ; P=.28178)$. However, a meta-regression coefficient was significantly negative for the proportion of men (coefficient, -0.00960 ; $95 \% \mathrm{CI},-0.01806$ to $-0.00114 ; P=.02621$; Figure 4 ), 


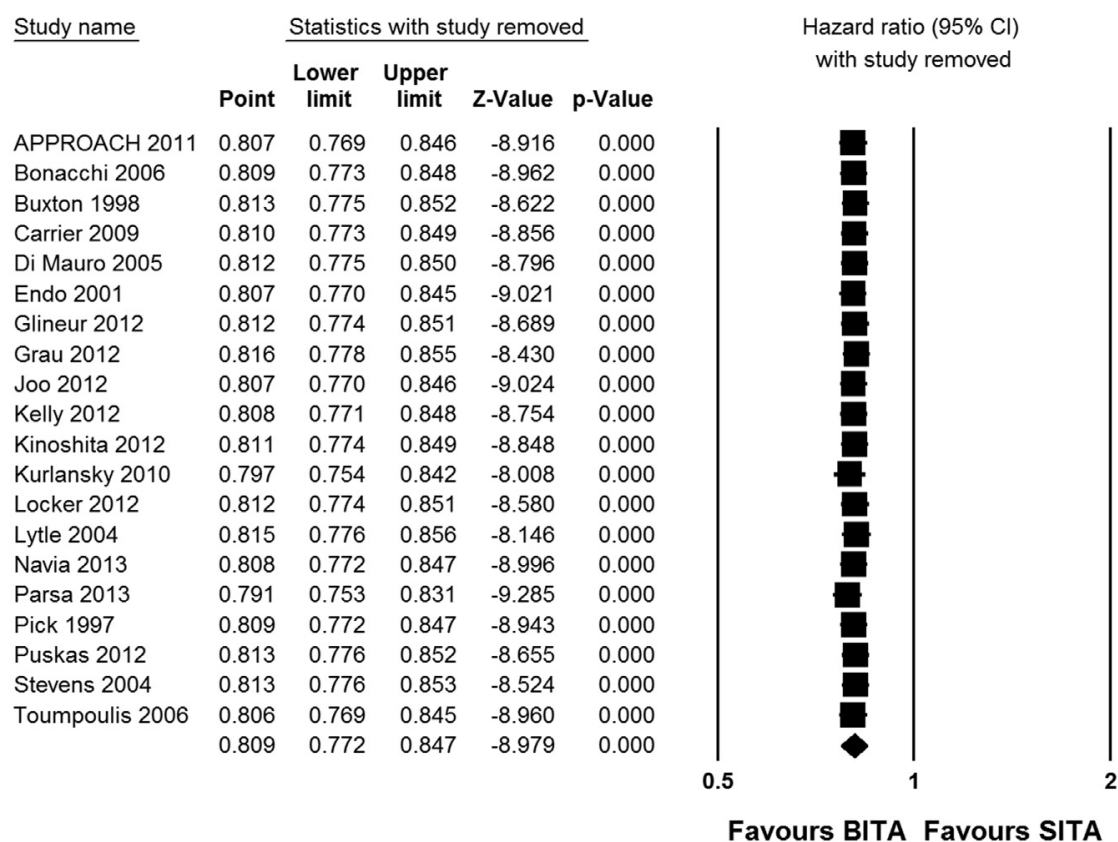

FIGURE 2. One-study-removed meta-analysis of adjusted hazard ratios for long-term mortality among patients assigned to bilateral versus single internal thoracic artery grafting. CI, Confidence interval; BITA, bilateral internal thoracic artery; SITA, single internal thoracic artery grafting.

which would indicate that as the proportion of men increases, the HR decreases; that is, BITA grafting is more beneficial in reducing long-term mortality.

\section{DISCUSSION}

The results of our analysis suggest that BITA grafting may reduce long-term mortality by $20 \%$ relative to SITA

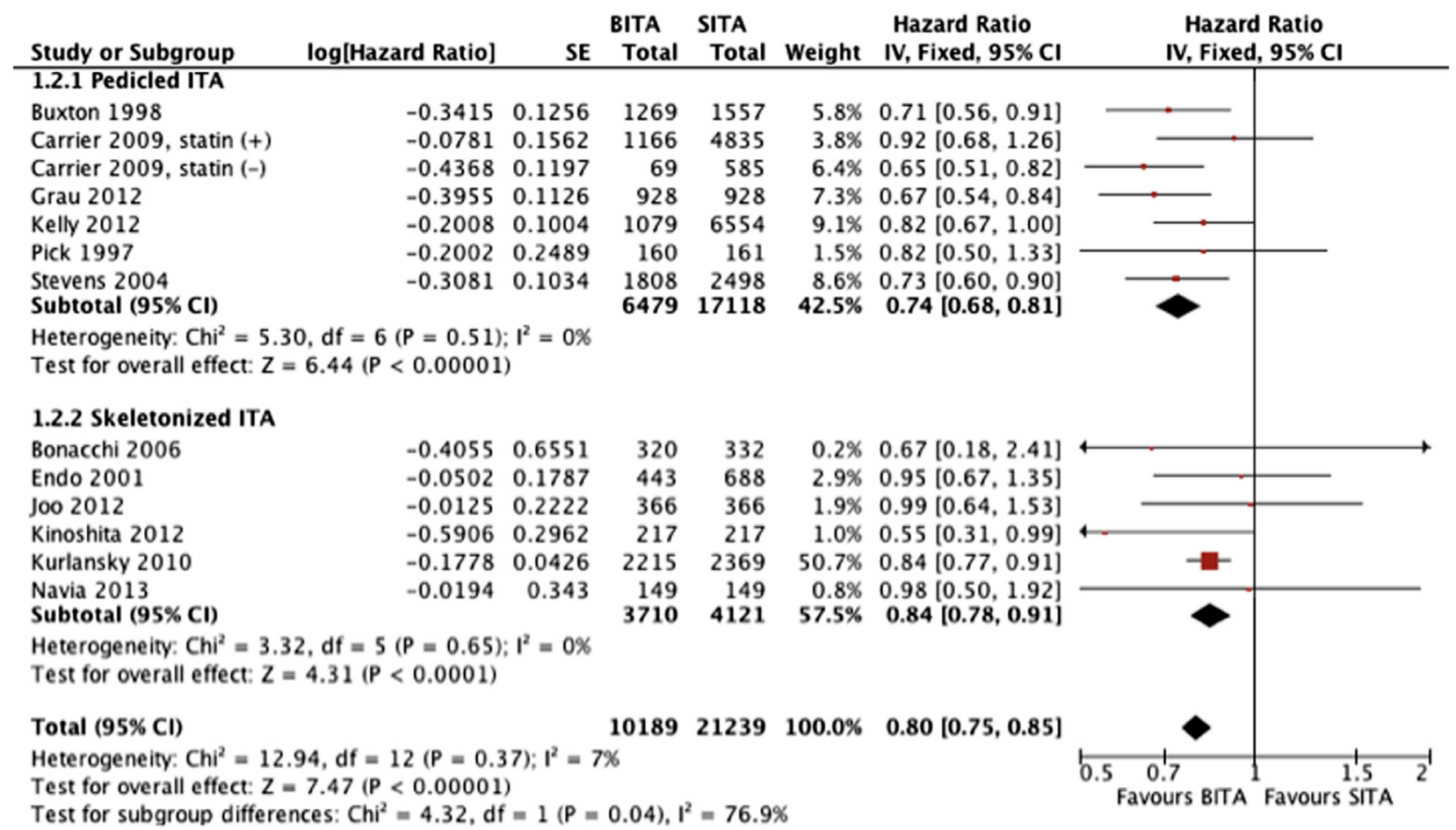

FIGURE 3. Forest plot of adjusted hazard ratios for long-term mortality among patients assigned to bilateral versus single internal thoracic artery grafting pooled separately in studies using the pedicled and skeletonized internal thoracic artery. BITA, Bilateral internal thoracic artery; SITA, single internal thoracic artery grafting; $S E$, standard error; $I V$, inverse variance; $C I$, confidence interval; ITA, internal thoracic artery. 


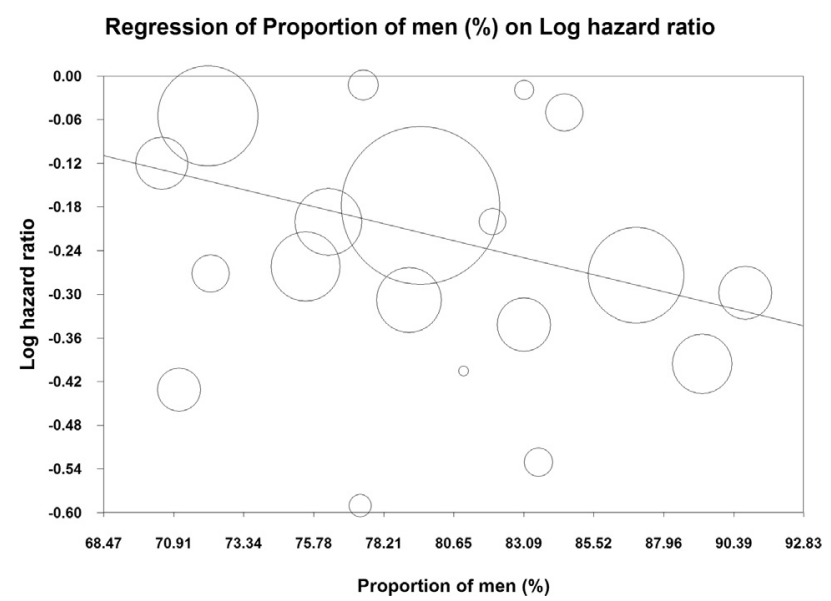

FIGURE 4. Meta-regression of proportion of men (\%) on logarithmic hazard ratio for long-term mortality among patients assigned to bilateral versus single internal thoracic artery grafting.

grafting in patients undergoing $\mathrm{CABG}$, which is most compelling with adjusted data from 70,897 patients assigned in 20 different observational studies and robust in sensitivity analyses. Because the present meta-analysis overcomes the limitations of the analysis Taggart and colleagues ${ }^{1}$ mentioned earlier, the results could provide better (not the best) evidence until a large randomized controlled trial reports long-term outcomes in the future.

In 1 of our sensitivity analyses, BITA grafting was associated with a $26 \%$ reduction in long-term mortality relative to SITA grafting in pedicled ITA studies, which was better ( $P$ for subgroup differences $=.04$ ) than a $16 \%$ reduction in skeletonized ITA studies. The 6 skeletonized ITA studies $^{13,16,19,21,22,25}$ analyzed enrolled only one-third (approximately 8000 patients) of approximately 24,000 patients enrolled in the 6 pedicled ITA studies. $3,8,14,18,20,27$ Evidence is limited in terms of pedicled versus skeletonized ITA-graft patency. ${ }^{29}$ Although theoretically skeletonization of the ITA might adversely affect its longterm resistance to atherosclerosis, the skeletonized ITA has not been used long enough to establish whether a decline in patency will occur after several years. ${ }^{30}$ In a recent systematic review, the follow-up data were not long-term; only 2 studies provided patency rates beyond 5 years. ${ }^{29}$ Also, only 4 were comparative studies of skeletonized versus pedicled conduits, in which skeletonization patency was at least comparable with pedicled conduits and in 2 studies even higher. ${ }^{29}$ Sternal wound infection (SWI), the most serious manifestation of which is mediastinitis, is recognized as an important complication of CABG, and mediastinitis can be severe and potentially lethal. Skeletonized ITA grafting seems to reduce the incidence of postoperative SWI in comparison with pedicled ITA grafting after CABG. A recent meta-analysis ${ }^{31}$ of 22 studies (involving 4817 patients) showed a statistically significant difference in favor of skeletonized ITA grafting (odds ratio [OR], 0.443; $95 \% \mathrm{CI}, 0.323$ to $0.608, P<.001$ ), which was also observed in subgroups of BITA grafting (OR, 0.381; 95\% CI, 0.257 to $0.565 ; P<.001)$ and diabetic patients with BITA grafting (OR, $0.188 ; 95 \%$ CI, 0.098 to $0.360 ; P<.001$ ). To confirm the long-term survival benefit of BITA grafting in pedicled versus skeletonized ITA studies suggested in our sensitivity analysis, further investigations are required.

One of our meta-regression analyses indicated that as the proportion of men increases, BITA grafting is more beneficial in reducing long-term mortality. A post hoc analysis ${ }^{32}$ of the same cohort (4584 patients) as the study by Kurlansky and colleagues ${ }^{22}$ (included in the present meta-analysis) may support our result. In multivariable Cox proportional hazard regression analyses for unmatched patients, the choice of the BITA conduit was identified as a predictor of late survival among men (HR, $0.8 ; 95 \% \mathrm{CI}, 0.7$ to 0.9 ; $P<.001)$ but not associated with late survival among women. ${ }^{32}$ In the propensity score matched male groups, the median survival for SITA patients was 14.2 years (95\% CI, 13.2 to 15.2$)$ compared with 15.8 years $(95 \%$ CI, 15.0 to 16.8 ) for BITA patients. The equality of the survival distribution for these 2 groups of male patients demonstrated a significant difference $(P<.001){ }^{32}$ These results provide further evidence of the survival benefits achieved in male patients with BITA grafting. In the propensity score matched female groups, however, the median survival for SITA patients was 14.0 years (95\% CI, 12.8 to 15.4$)$ and that for BITA patients was 13.7 years $(95 \% \mathrm{CI}, 12.8$ to 14.7), with no significant difference in survival distribution $(P=.571) .{ }^{32}$ Therefore, BITA grafting seems to confer an incremental survival benefit relative to SITA grafting in men but not in women. Although these results represent the largest experience in the current literature that specifically addresses long-term survival of BITA grafting in women, it may be that 329 patients, compared with the male cohorts of more than 1000 patients, is just not sufficient to demonstrate a survival advantage (type II error). ${ }^{32}$

As an important point of concern, SWI is one of the main reasons limiting the extensive use of more than 1 ITA. A recent meta-analysis ${ }^{33}$ of 32 studies (consisting of 172,880 patients) showed that BITA grafting increases the risk of SWI compared with SITA grafting. The risk of SWI in the BITA group was higher (RR for SITA vs BITA, $0.62 ; 95 \%$ CI, 0.55 to 0.71 ) than that in the SITA group. This adverse effect further extends to diabetic and elderly patients ${ }^{33}$; BITA grafting was also associated with a higher risk of SWI in diabetic patients (RR for SITA vs BITA, $0.65 ; 95 \% \mathrm{CI}, 0.52$ to $0.81 ; 12$ studies consisting of 128,109 patients) as well as elderly patients (RR for SITA vs BITA, $0.45 ; 95 \%$ CI, 0.33 to $0.62 ; 5$ studies consisting of 8206 patients). Also in another recent meta-anal$\mathrm{ysis}^{34}$ of 1 randomized controlled trial and 10 observational 
studies (enrolling 126,235 diabetic patients), deep SWI occurred in $3.1 \%$ and $1.6 \%$ for the BITA and LITA cohorts, respectively (RR, $1.71 ; 95 \% \mathrm{CI}, 1.37$ to 2.14 ). Despite the higher risk of SWI in BITA grafting, the present metaanalysis demonstrated the obvious benefit for BITA grafting for long-term survival.

Our analysis must be viewed in the context of its limitations. We used only data from observational studies, not randomized controlled trials. Although patients enrolled in randomized trials may not be representative of patients typically seen in clinical practice, this is the study design least vulnerable to bias because randomized trials balance both known and unknown confounders across treatment groups. Potential biases are likely to be greater for observational studies compared with randomized trials, however, so results should always be interpreted with caution when they are included in reviews and meta-analyses. ${ }^{35}$ Particular concerns arise with respect to differences between patients in different intervention groups (selection bias). Unlike for randomized trials, it would usually be appropriate to analyze adjusted (rather than unadjusted) effect estimates, that is, analyses that attempt to control for confounding. ${ }^{3.3}$ To reduce the effect of treatment selection bias and potential confounding in observational studies, rigorous adjustment for significant differences in the baseline characteristics of patients should be conducted. Furthermore, adjusted (not unadjusted) estimates ought to be pooled in a metaanalysis that includes observational studies. In the present meta-analysis, we strictly abstracted, then combined in a meta-analysis, exclusive adjusted (not unadjusted) risk estimates from observational studies. Meanwhile, our results may be influenced by a publication bias favoring BITA grafting. This risk was minimized through an exhaustive search of the available literature. Although the statistical tests did not indicate publication bias, there is clearly limited power to detect such bias, given the small number of studies examined.

Despite these acknowledged limitations, we found that, based on an updated meta-analysis of exclusive adjusted risk estimates from 20 observational studies enrolling more than 70,000 patients, BITA grafting is likely effective in the prevention of long-term mortality in patients undergoing CABG. Because mortality reduction must imply the greatest clinical benefit among patients undergoing CABG, BITA grafting should be considered for patients who meet the criteria for enrollment in the observational studies discussed earlier.

\section{References}

1. Taggart DP, D'Amico R, Altman DG. Effect of arterial revascularisation on survival: a systematic review of studies comparing bilateral and single internal mammary arteries. Lancet. 2001;358:870-5.

2. Berreklouw E, Schönberger JP, Ercan H, Koldewijn EL, de Bock M, Verwaal VJ, et al. Does it make sense to use two internal thoracic arteries? Ann Thorac Surg. 1995;59:1456-63.
3. Buxton BF, Komeda M, Fuller JA, Gordon I. Bilateral internal thoracic artery grafting may improve outcome of coronary artery surgery. Risk-adjusted survival. Circulation. 1998;98(19 Suppl):II1-6.

4. Dewar LR, Jamieson WR, Janusz MT, Adeli-Sardo M, Germann E, MacNab JS, et al. Unilateral versus bilateral internal mammary revascularization. Survival and event-free performance. Circulation. 1995;92(9 Suppl):II8-13.

5. Lytle BW, Blackstone EH, Loop FD, Houghtaling PL, Arnold JH, Akhrass R, et al. Two internal thoracic artery grafts are better than one. J Thorac Cardiovasc Surg. 1999;117:855-72.

6. Morris JJ, Smith LR, Glower DD, Muhlbaier LH, Reves JG, Wechsler AS, et al. Clinical evaluation of single versus multiple mammary artery bypass. Circula tion. 1990;82(5 Suppl):IV214-23.

7. Naunheim KS, Barner HB, Fiore AC. 1990: Results of internal thoracic artery grafting over 15 years: single versus double grafts. 1992 update. Ann Thorac Surg. 1992;53:716-8.

8. Pick AW, Orszulak TA, Anderson BJ, Schaff HV. Single versus bilateral internal mammary artery grafts: 10-year outcome analysis. Ann Thorac Surg. 1997;64 599-605.

9. Parsa CJ, Shaw LK, Rankin JS, Daneshmand MA, Gaca JG, Milano CA, et al Twenty-five-year outcomes after multiple internal thoracic artery bypass. J Thorac Cardiovasc Surg. 2013;145:970-5.

10. Myers WO, Berg R, Ray JF, Douglas-Jones JW, Maki HS, Ulmer RH, et al All-artery multigraft coronary artery bypass grafting with only internal thoracic arteries possible and safe: a randomized trial. Surgery. 2000;128:650-9.

11. Taggart DP, Altman DG, Gray AM, Lees B, Nugara F, Yu LM, et al; ART Investigators. Randomized trial to compare bilateral vs. single internal mammary coronary artery bypass grafting: 1-year results of the Arterial Revascularisation Trial (ART). Eur Heart J. 2010;31:2470-81.

12. Kieser TM, Lewin AM, Graham MM, Martin BJ, Galbraith PD, Rabi DM, et al; APPROACH Investigators. Outcomes associated with bilateral internal thoracic artery grafting: the importance of age. Ann Thorac Surg. 2011;92:1269-75; discussion 1275-6.

13. Bonacchi M, Maiani M, Prifti E, Di Eusanio G, Di Eusanio M, Leacche M. Urgent/emergent surgical revascularization in unstable angina: influence of different type of conduits. J Cardiovasc Surg (Torino). 2006;47:201-10.

14. Carrier M, Cossette M, Pellerin M, Hébert Y, Bouchard D, Cartier R, et al. Statin treatment equalizes long-term survival between patients with single and bilateral internal thoracic artery grafts. Ann Thorac Surg. 2009;88:789-95; discussion 795.

15. Di Mauro M, Iacò AL, Contini M, Vitolla G, Weltert L, Di Giammarco G, et al First time myocardial revascularization in patients younger than 70 years. Single versus double internal mammary artery. Ital Heart J. 2005;6:390-5.

16. Endo M, Nishida H, Tomizawa Y, Kasanuki H. Benefit of bilateral over single internal mammary artery grafts for multiple coronary artery bypass grafting. Circulation. 2001;104:2164-70

17. Glineur D, D’hoore W, Price J, Dorméus S, de Kerchove L, Dion R, et al. Survival benefit of multiple arterial grafting in a 25-year single-institutional experience: the importance of the third arterial graft. Eur J Cardiothorac Surg. 2012;42: 284-90; discussion 290-1.

18. Grau JB, Ferrari G, Mak AW, Shaw RE, Brizzio ME, Mindich BP, et al. Propensity matched analysis of bilateral internal mammary artery versus single left internal mammary artery grafting at 17-year follow-up: validation of a contemporary surgical experience. Eur J Cardiothorac Surg. 2012;41:770-5; discussion 776.

19. Joo HC, Youn YN, Yi G, Chang BC, Yoo KJ. Off-pump bilateral internal thoracic artery grafting in right internal thoracic artery to right coronary system. Ann Thorac Surg. 2012;94:717-24.

20. Kelly R, Buth KJ, Légaré JF. Bilateral internal thoracic artery grafting is superior to other forms of multiple arterial grafting in providing survival benefit after coronary bypass surgery. J Thorac Cardiovasc Surg. 2012;144:1408-15.

21. Kinoshita T, Asai T, Suzuki T, Kuroyanagi S, Hosoba S, Takashima N. Off-pump bilateral skeletonized internal thoracic artery grafting in elderly patients. Ann Thorac Surg. 2012;93:531-6.

22. Kurlansky PA, Traad EA, Dorman MJ, Galbut DL, Zucker M, Ebra G. Thirtyyear follow-up defines survival benefit for second internal mammary artery in propensity-matched groups. Ann Thorac Surg. 2010;90:101-8.

23. Locker C, Schaff HV, Dearani JA, Joyce LD, Park SJ, Burkhart HM, et al. Multiple arterial grafts improve late survival of patients undergoing coronary artery bypass graft surgery: analysis of 8622 patients with multivessel disease. Circula tion. 2012;126:1023-30.

24. Lytle BW, Blackstone EH, Sabik JF, Houghtaling P, Loop FD, Cosgrove DM. The effect of bilateral internal thoracic artery grafting on survival during 20 postoperative years. Ann Thorac Surg. 2004;78:2005-12; discussion 2012-4. 
25. Navia D, Vrancic M, Piccinini F, Camporrotondo M, Thierer J, Gil C, et al. Is the second internal thoracic artery better than the radial artery in total arterial offpump coronary artery bypass grafting? A propensity score-matched follow-up study. J Thorac Cardiovasc Surg. 2014;147:632-8.

26. Puskas JD, Sadiq A, Vassiliades TA, Kilgo PD, Lattouf OM. Bilateral internal thoracic artery grafting is associated with significantly improved long-term survival, even among diabetic patients. Ann Thorac Surg. 2012;94:710-5; discussion 715-16.

27. Stevens LM, Carrier M, Perrault LP, Hébert Y, Cartier R, Bouchard D, et al. Single versus bilateral internal thoracic artery grafts with concomitant saphenous vein grafts for multivessel coronary artery bypass grafting: effects on mortality and event-free survival. J Thorac Cardiovasc Surg. 2004;127:1408-15.

28. Toumpoulis IK, Anagnostopoulos CE, Balaram S, Swistel DG, Ashton RC Jr, DeRose JJ Jr. Does bilateral internal thoracic artery grafting increase long-term survival of diabetic patients? Ann Thorac Surg. 2006;81:599-606; discussion 606-7.

29. Ali E, Saso S, Ashrafian H, Athanasiou T. Does a skeletonized or pedicled left internal thoracic artery give the best graft patency? Interact Cardiovasc Thorac Surg. 2010;10:97-104.

30. Del Campo C. Pedicled or skeletonized? A review of the internal thoracic artery graft. Tex Heart Inst J. 2003;30:170-5.
31. Sá MP, Ferraz PE, Escobar RR, Vasconcelos FP, Ferraz AA, Braile DM, et al. Skeletonized versus pedicled internal thoracic artery and risk of sternal wound infection after coronary bypass surgery: meta-analysis and metaregression of 4817 patients. Interact Cardiovasc Thorac Surg. 2013;16: 849-57.

32. Kurlansky PA, Traad EA, Dorman MJ, Galbut DL, Zucker M, Ebra G. Bilateral internal mammary artery grafting reverses the negative influence of gender on outcomes of coronary artery bypass grafting surgery. Eur J Cardiothorac Surg. 2013;44:54-63.

33. Dai C, Lu Z, Zhu H, Xue S, Lian F. Bilateral internal mammary artery grafting and risk of sternal wound infection: evidence from observational studies. Ann Thorac Surg. 2013;95:1938-45.

34. Deo SV, Shah IK, Dunlay SM, Erwin PJ, Locker C, Altarabsheh SE, et al. Bilateral internal thoracic artery harvest and deep sternal wound infection in diabetic patients. Ann Thorac Surg. 2013;95:862-9.

35. Reeves BC, Deeks JJ, Higgins JP, Wells GA. Including non-randomized studies. In: Higgins JP, Green S, eds. Cochrane Handbook for Systematic Reviews of Interventions Version 5.1.0 (updated March 2011). The Cochrane Collaboration; 2011, Chapter 13. Available at: www.cochrane-handbook.org. 